\title{
UMA ANÁLISE DE FATORES NA IMPLEMENTAÇÃO DOS PRINCÍPIOS DE GESTÃO DA QUALIDADE DA SÉRIE DE NORMAS ISO 9000 EM UMA ORGANIZAÇÃO AGROINDUSTRIAL
}

\section{AN FACTORS ANALYSIS IN IMPLEMENTATION OF QUALITY MANAGEMENT PRINCIPLES OF ISO 9000 STANDARDS IN AN AGRO-INDUSTRIAL ORGANIZATION}

\author{
André Luiz Corrêa - andreluizcorrea@outlook.com \\ Mestrando em Administração pela UNESP - Jaboticabal - SP - Brasil \\ Profa. Dra. Glaucia Aparecida Prates - glaucia@itapeva.unesp.br \\ Universidade Estadual Paulista - UNESP - Itapeva - SP - Brasil \\ Profa. Dra. Lesley Carina do Lago Attadia Galli - lesleyattadia@ fcav.unesp.br \\ Universidade Estadual Paulista - UNESP - Jaboticabal - SP - Brasil \\ Prof. Dr. Antonio Francisco Savi - savi@itapeva.unesp.br \\ Universidade Estadual Paulista - UNESP - Itapeva - SP - Brasil

\section{RESUMO}

O objetivo desta pesquisa foi identificar em que grau os princípios de gestão da qualidade da série de normas ISO 9000 estão inter-relacionados em uma organização agroindustrial. Além disso, este estudo busca reduzir o conjunto inicial de vinte e uma variáveis para poucas variáveis que expliquem como esses princípios de gestão estão inter-relacionados. Foi realizado um estudo de caso em uma organização agroindustrial certificada ISO 9001 envolvida na cadeia de suprimentos sucroenergética. $O$ instrumento de coleta de dados foi elaborado tendo como referência a norma ISO 10014. Foi calculado o coeficiente de Cronbach sendo obtido um valor de $\propto=0,94$, demonstrando forte consistência interna no questionário. Foram obtidas 98 respostas completas que foram analisadas por meio de técnicas estatísticas descritivas e multivariadas. Para as análises estatísticas foram utilizados os softwares Excel® 2016 e Statistica ${ }^{\circledR}$ 7.0. Por meio da análise de fatores as vinte e uma variáveis iniciais foram reduzidas a dois fatores, que foram denominados gestão de processos e gestão de pessoas. Conclui-se que os objetivos da pesquisa foram plenamente alcançados demonstrando a eficácia da aplicação de técnicas multivariadas para compreensão de fenômenos resultantes da ação de variáveis inter-relacionadas. A redução das vinte e uma variáveis iniciais para dois fatores distintos entre si possibilita ao gestor maior eficiência na tomada de decisões estabelecendo planos de ações mais assertivos.

Palavras-chave: Qualidade. ISO 9000. Multivariada. Princípios. 


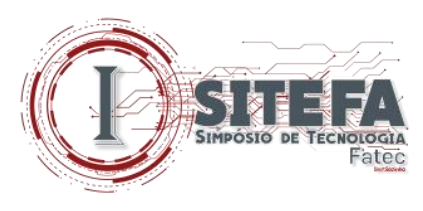

\begin{abstract}
The objective of this research was to identify the degree to which the quality management principles of the ISO 9000 series are interrelated in an agro-industrial organization. In addition, this study seeks to reduce the initial set of twenty-one variables to a few variables that explain how these management principles are interrelated. A case study was carried out in an ISO 9001 certified agroindustrial organization involved in the sugarcane supply chain. The data collection instrument was elaborated with ISO 10014 as a reference. The Cronbach coefficient was calculated and a value $\propto=0,94$ was obtained, showing a strong internal consistency in the questionnaire. 98 complete answers were obtained, which were analyzed using descriptive and multivariate statistical techniques. For the statistical analyzes, the software Excel® 2016 and Statistica ${ }^{\circledR} 7.0$ were used. Through the analysis of factors, the twenty-one initial variables were reduced to two factors, which were called process management and people management. It is concluded that the objectives of the research were fully achieved demonstrating the effectiveness of the application of multivariate techniques to understand phenomena resulting from the action of interrelated variables. The reduction of the twenty-one initial variables to two different factors allows the manager greater efficiency in decision-making by establishing more assertive action plans.
\end{abstract}

Keywords: Quality. ISO 9000 standards. Multivariate. Principles.

\title{
DOI:
}

\section{INTRODUÇÃO}

Se até algumas décadas passadas investir em gestão da qualidade podia ser considerado como uma estratégia que criava vantagens competitivas para as organizações, hoje o mesmo não pode ser dito. Cada vez mais as empresas têm buscado alcançar uma posição de destaque no ambiente competitivo globalizado (GOHR; FAUSTINO, 2017).

Para a implementação de um sistema de gestão da qualidade é recomendado que seja utilizado um modelo de referência (TOLEDO et al., 2012). Há diversos tipos de modelos de referência para a gestão da qualidade, dentre os quais destacam-se os modelos normativos, modelos corporativos, modelos de excelência de gestão e modelos teóricos propostos por autores (TOLEDO et al, 2012; SINGH; SMITH, 2006). Cabe à organização decidir-se por qual tipo de modelo de referência adotar, considerando, principalmente, as exigências de seus clientes e seus interesses estratégicos.

No contexto desta pesquisa o modelo de referência considerado está estabelecido na norma ABNT NBR ISO 9001:2015, que é um modelo de referência amplamente difundido é 


\section{(1) SIIEFA}

proposto pela entidade normativa ISO (COMITÊ BRASILEIRO DA QUALIDADE - CB-25, 2017; INTERNATIONAL ORGANIZATION FOR STANDARTZATION - ISO, 2017).

A pesquisa investiga em que grau os princípios de gestão da qualidade estabelecidos na norma ISO 9000 estão inter-relacionados e implementados, buscando reduzi-los a fatores latentes por meio da técnica estatística multivariada conhecida como análise de fatores.

\section{FUNDAMENTAÇÃO TEÓRICA}

A série de normas ISO 9000 é assim conhecida porque trata-se de um trio de normas: ISO 9000, ISO 9001 e ISO 9004. Cada uma dessas normas possui a sua especificidade.

A norma ISO 9000 apresenta os princípios norteadores de gestão da qualidade para a série de normas (ABNT, 2015a). A norma ISO 9001 apresenta requisitos para que um sistema de gestão da qualidade seja implementado e mantido de forma eficaz (ABNT, 2015b). Enquanto a ISO 9001 possui como foco alcançar a satisfação do cliente por meio da eficácia do sistema do gestão da qualidade, a ISO 9004 tem um foco mais abrangente, pois essa norma propõe que as ações executadas levem em consideração além dos clientes, a satifação dos acionistas, empregados, sociedade, fornecedores etc (ABNT, 2010).

\subsection{Princípios de gestão da qualidade e benefícios de sua implementação}

Segundo a ABNT (2015a, p.1), os princípios de gestão da qualidade "[...] fornecem à organização a capacidade para enfrentar os desafios apresentados por um ambiente que é profundamente diferente a partir das últimas décadas”. A norma ISO 9000 sustenta ainda que no contexto atual, caracterizado pela mudança acelerada, globalização dos mercados e o advento do conhecimento como principal recurso o impacto da qualidade se estende para além da satisfação do cliente, podendo impactar diretamente a reputação da organização (ABNT, 2015a).

Segundo a ABNT (2015a), os princípios de gestão da qualidade são foco no cliente, liderança, engajamento das pessoas, abordagem de processo, melhoria, tomada de decisão baseada em evidência e gestão do relacionamento. Os princípios da gestão da qualidade são 


\section{(1) $10=0$}

inter-relacionados e nenhum princípio individual pode ser considerado mais importante do que outro (ABNT, 2015a).

A aplicação dos princípios de gestão da qualidade estabelecidos na norma ISO 9000 contribui para que o sistema de gestão da qualidade implementado alcance melhores resultados, o que, por sua vez, leva à organização a melhores resultados (FONSECA e DOMINGUES, 2017). Mas, como esses princípios não se constituem de requisitos eles não podem ser implementados diretamente nos processos de negócio. Entretanto, os benefícios da adoção dos princípios de gestão da qualidade podem ser obtidos pela aplicação dos requisitos para um sistema de gestão da qualidade estabelecidos na norma ISO 9001 (ABNT, 2015b).

Rusjan e Alič (2010), em sua pesquisa empírica, associaram os benefícios obtidos pelas organizações por meio da implementação da ISO 9001 com as quatro dimensões de indicadores de desempenho do método de planejamento estratégico conhecido como Balanced Scorecard. Wolniak (2011), em sua pesquisa com mais de 750 empresas polonesas com sistemas de gestão da qualidade estruturados conforme os requisitos da ISO 9001, identificou benefícios econômicos e mercadológicos obtidos, considerando a perspectiva da norma ISO 10014.

A utilização da ISO 10014 para análise dos benefícios percebidos pela aplicação dos princípios de gestão da qualidade também foi realizada por Moradi et al. (2015) em seu trabalho sobre níveis de maturidade de organizações hospitalares. Denton e Maatgi (2016), em sua proposta de um modelo de ambiente de trabalho orientado para o sucesso na implementação das normas ISO 9000, utilizam a ISO 10014 e a ISO 9004 para análise da maturidade do sistema de gestão da qualidade das organizações.

\subsection{Análise de fatores por componentes principais}

A análise de fatores por componentes principais busca reduzir o espaço multidimensional de um problema de pesquisa para poucas dimensões que expliquem o maior número possível de informações disponíveis do objeto em estudo (LOESCH e HOELTGEBAUM, 2012; LATTIN, CARROLL e GREEN, 2011; ARANHA e ZAMBALDI, 2008). Essa técnica busca reduzir o espaço de variáveis criando eixos ortogonais que são combinações lineares das variáveis originais denominados componentes principais. Esta 


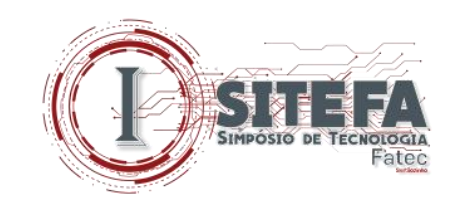

técnica envolve um procedimento matemático que transforma um número de variáveis possivelmente correlacionadas em um número menor de variáveis não correlacionadas denominadas componentes principais.

A análise por componentes principais é o método mais utilizado para determinação de fatores (FÁVERO; BELFIORE; 2015). Kaiser (1958) propôs que fatores que possuam um autovalor maior que 1 podem ser considerados relevantes, pois geram componentes com quantidade relevante de informação das variáveis originais. Abaixo de 1 a quantidade de informação retida no componente não é relevante. Entretanto ao obter os fatores originais, suas disposições geometricamente no espaço fatorial são arbitrárias, normalmente não sendo possível distinguir claramente cargas fortes de cargas fracas nos fatores. Nesse sentido, procura-se promover a rotação desses fatores de modo a levarem-nos a posições que separem cargas altas de cargas baixas em mais de um fator. Vários métodos de rotação existem na literatura, sendo os mais utilizados, os métodos Varimax, Quartimax, Biquartimax e Equamax (HAIR et al., 2009).

\section{MATERIAIS E MÉTODOS}

O instrumento de coleta dados constituiu-se de um questionário elaborado tendo como referência a avaliação inicial do grau de percepção de benefícios resultantes pela aplicação dos princípios de gestão da qualidade proposto pela norma ISO 10014. O questionário apresentou vinte e umas questões (três para cada princípio de gestão da qualidade) com uma escala de respostas de 0 a 10, baseada na escala Likert.

Para validar o uso do questionário proposto, foi utilizado o coeficiente conhecido como alfa de Cronbach (CRONBACH, 1951). Essa checagem averigua a consistência interna das questões que compõem o grupo de variáveis (LOESCH; HOELTGEBAUM, 2012). O coeficiente alfa de Cronbach varia entre 0 e 1 , sendo que quanto mais próximo de 1 , melhor é a consistência interna do questionário. Apesar do alfa de Cronbach receber críticas quanto a sua escolha como método de consistência interna de questionários (MAROCO; GARCIAMARQUES, 2006) é um método amplamente utilizado. 


\section{(1) SITEFA}

O questionário foi aplicado por meio da ferramenta Google Forms®. Os dados coletados foram transferidos para planilhas eletrônicas do software Microsoft Excel@ para as análises descritivas e posteriormente para o software Statistica ${ }^{\circledR} 7.0$ para a análise de fatores.

Foi realizado um levantamento na base de dados do INMETRO de organizações industriais certificadas ISO 9001 e que estavam inseridas na cadeia de suprimentos do agronegócio sucroenergético no polo industrial do município de Sertãozinho, localizado na região de Ribeirão Preto, Estado de São Paulo.

O levantamento inicial apontou trinta e dois certificados ISO 9001 emitidos para as indústrias transformadoras no município de Sertãozinho. É importante salientar que o número de certificados emitidos não corresponde necessariamente ao número de organizações certificadas, visto que uma organização pode possuir mais que uma certificação para diferentes unidades de produção ou diferentes escopos de produção e de produtos.

Sendo assim, o número de organizações industriais certificadas constituiu-se em um total de vinte e sete empresas. Como a análise de dados da pesquisa utilizou técnicas multivariadas, que precisam de um número significativo de casos ou respondentes para as análises, focou-se em organizações com pelo menos cento e cinquenta empregados. Outros critérios restritivos foram utilizados: as organizações precisavam estar economicamente ativas e deveriam ser fornecedoras de bens de capital para usinas ou destilarias, produtoras de açúcar, etanol, energia e outros derivados.

Como resultante desses critérios para seleção dos casos a serem estudados, restou um total de seis organizações, que foram convidadas a participar da pesquisa. Apenas uma dessas organizações aceitou participar da pesquisa, solicitando que sua identidade fosse mantida em sigilo. A referida organização foi tratada na pesquisa como EMPRESA ALFA.

\section{RESULTADOS E DISCUSSÃO}

A EMPRESA ALFA foi fundada na primeira metade da década de 1980, no município de Sertãozinho, conhecido polo industrial sucroenergético na região metropolitana de Ribeirão Preto, no interior do Estado de São Paulo, com o objetivo inicial de prestação de serviços em assistência técnica, nas áreas de instrumentação e controle eletrônicos de processos aplicáveis às indústrias do setor sucroalcooleiro do interior paulista. Atualmente a 


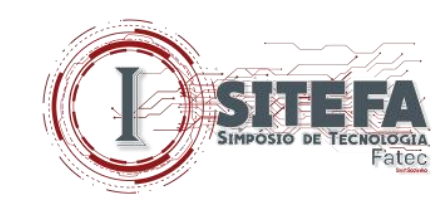

organização dispõe de quadro de aproximadamente 200 empregados diretos e indiretos e mantém sua certificação ISO 9001.

A pesquisa foi disponibilizada para os empregados da EMPRESA ALFA. Do total de 182 empregados direta ou indiretamente vinculados, foram obtidas 98 respostas completas e validadas. A Figura 1 sintetiza o perfil dos respondentes por área de atuação, nível hierárquico e tempo de atuação na empresa.

Figura 1 - Etapas da pesquisa

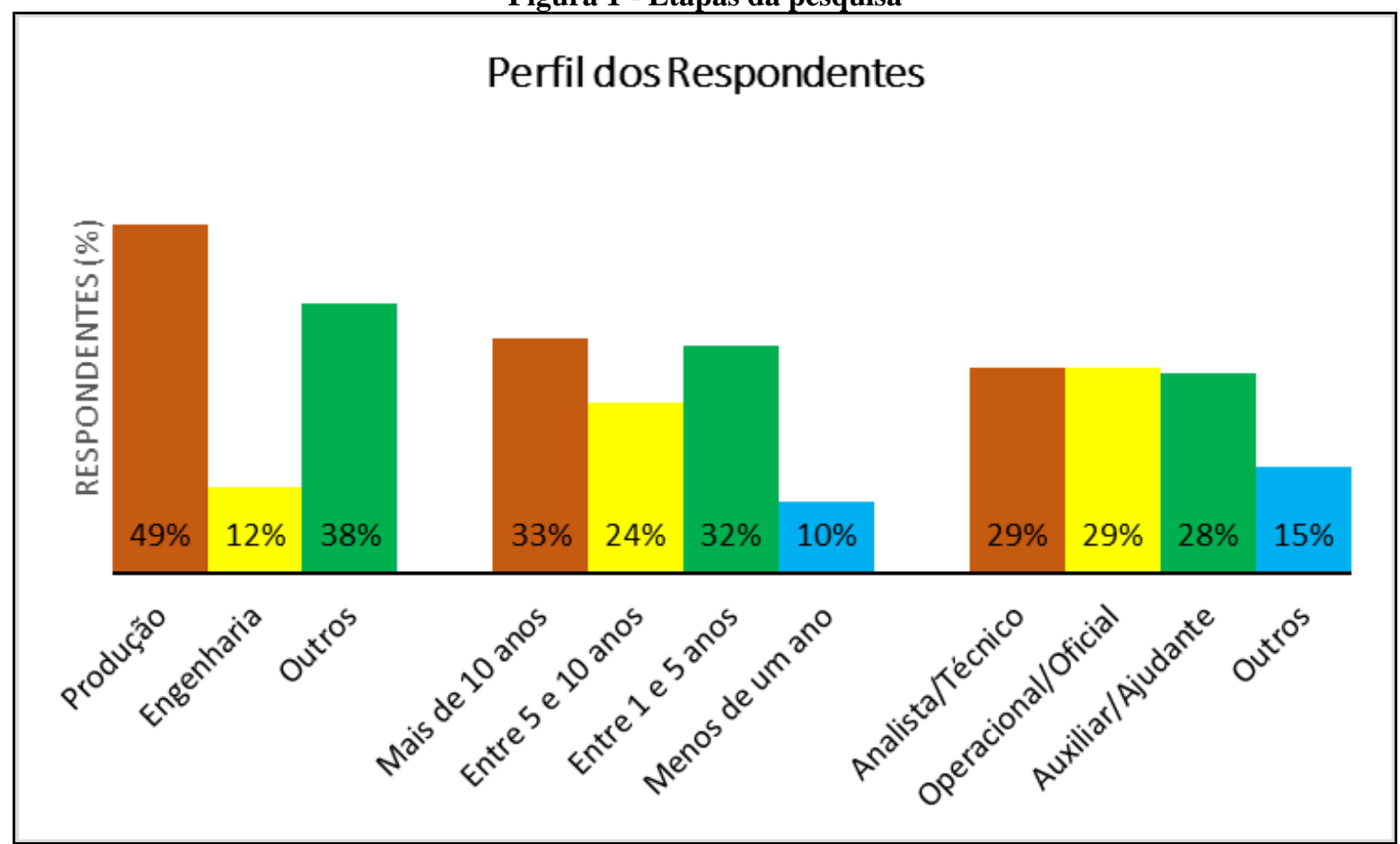

Fonte: elaborada pelos autores (2018).

Para cada princípio de gestão foram apresentadas três questões, totalizando 21 questões. As vinte e uma questões podem ser consideradas como 21 variáveis estatísticas numéricas. Cada questão pode ser avaliada em uma escala de 0 a 10 pontos, sendo que notas mais próximas de zero significavam discordância da afirmação e notas mais próximas de dez significavam maior concordância com a afirmação. As questões foram aleatoriamente distribuídas para que o respondente não identificasse qual princípio de gestão da qualidade cada questão pretendia avaliar.

A Tabela 1 apresenta a média, a variância e o desvio-padrão obtidos na pesquisa para cada variável observada. Observa-se que as médias oscilam ente 5 e 6 , o que demonstra, $a$ priori, espaço para ações para a melhoria da percepção dos benefícios resultantes da 


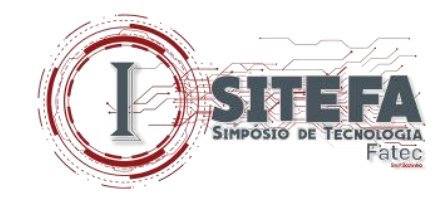

implementação dos princípios de gestão da qualidade. O princípio de gestão da qualidade foco no cliente é o mais bem avaliado pelos empregados, sendo que de outro modo, o princípio de gestão liderança é o que possui pior avaliação média.

Tabela 1 - Medidas de tendência central das variáveis observadas

\begin{tabular}{ccccc}
\hline Variáveis & $\begin{array}{c}\text { Média geral do } \\
\text { princípio }\end{array}$ & Média da variável & Variância & Desvio-padrão \\
\hline FC1 & & 6,592 & 3,048 & 1,746 \\
FC2 & 6,313 & 5,918 & 4,241 & 2,059 \\
FC3 & 6,429 & 4,124 & 2,031 \\
\hline L1 & 5,449 & 7,013 & 2,648 \\
L2 & 5,429 & 5,163 & 6,406 & 2,531 \\
L3 & 5,673 & 5,789 & 2,406 \\
\hline EP1 & 5,286 & 7,216 & 2,686 \\
EP2 & 6,088 & 6,000 & 6,351 & 2,520 \\
EP3 & & 6,224 & 2,453 & 1,566 \\
\hline AP1 & 6,122 & 3,887 & 1,972 \\
AP2 & 5,735 & 5,841 & 2,417 \\
AP3 & 6,027 & 5,429 & 6,053 & 2,460 \\
\hline M1 & & 5,443 & 2,333 \\
M2 & & 6,020 & 4,927 & 2,220 \\
M3 & 5,701 & 5,653 & 5,012 & 2,239 \\
\hline TD1 & & 5,776 & 4,506 & 2,123 \\
TD2 & 5,714 & 5,856 & 2,420 \\
TD3 & 5,741 & 5,735 & 4,321 & 2,079 \\
\hline GR1 & & 6,102 & 4,752 & 2,180 \\
GR2 & 5,810 & 5,673 & 6,531 & 2,556 \\
GR3 & & 5,653 & 5,342 & 2,311 \\
\hline
\end{tabular}

Fonte: elaborada pelos autores (2018).

A Tabela 2 apresenta a correlação geral entre os dados padronizados das vinte e uma variáveis analisadas na pesquisa. Considerando Hair et al (2009) e os interesses dessa pesquisa, foram definidas como relevantes correlações a partir de 0,65. Observa-se que a variável EP3 correlaciona-se apenas com as variáveis L3 e AP1, não se correlacionando significativamente com nenhuma outra variável, inclusive com as variáveis de seu próprio grupo (EP1 e EP2). Sendo assim, a variável EP3 foi retirada da posterior análise multivariada. As demais variáveis correlacionam-se significativamente com as variáveis de seus grupos e 


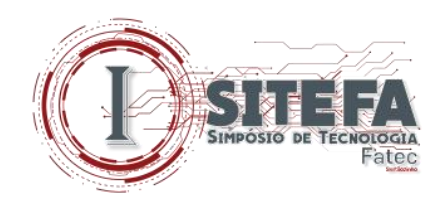

apresentam correlações significativas com ao menos uma variável de outro grupo e foram mantidas para a análise de fatores.

Tabela 2 - Coeficientes de correlação entre as variáveis padronizadas

\begin{tabular}{|c|c|c|c|c|c|c|c|c|c|c|c|c|c|c|c|c|c|c|c|c|c|}
\hline & $F C 1$ & $F C 2$ & $F C 3$ & $L 1$ & $L 2$ & L3 & $E P 1$ & $E P 2$ & EP3 & $A P 1$ & $A P 2$ & $A P 3$ & $M 1$ & $M 2$ & M3 & $T D 1$ & $T D 2$ & TD3 & GRI & $G R 2$ & GR3 \\
\hline $\mathrm{FC1}$ & 1,000 & & & & & & & & & & & & & & & & & & & & \\
\hline $\mathrm{FC} 2$ & 0,839 & 1,000 & & & & & & & & & & & & & & & & & & & \\
\hline FC3 & 0,748 & 0,694 & 1,000 & & & & & & & & & & & & & & & & & & \\
\hline L1 & 0,727 & 0,797 & 0,558 & 1,000 & & & & & & & & & & & & & & & & & \\
\hline L2 & 0,710 & 0,798 & 0,568 & 0,906 & 1,000 & & & & & & & & & & & & & & & & \\
\hline L3 & 0,758 & 0,802 & 0,818 & 0,748 & 0,720 & 1,000 & & & & & & & & & & & & & & & \\
\hline EP1 & 0,737 & 0,765 & 0,635 & 0,892 & 0,933 & 0,745 & 1,000 & & & & & & & & & & & & & & \\
\hline EP2 & 0,661 & 0,667 & 0,516 & 0,775 & 0,798 & 0,643 & 0,758 & 1,000 & & & & & & & & & & & & & \\
\hline EP3 & 0,645 & 0,594 & 0,593 & 0,365 & 0,323 & 0,671 & 0,374 & 0,413 & 1,000 & & & & & & & & & & & & \\
\hline AP1 & 0,746 & 0,822 & 0,604 & 0,672 & 0,629 & 0,781 & 0,626 & 0,602 & 0,703 & 1,000 & & & & & & & & & & & \\
\hline AP2 & 0,804 & 0,872 & 0,758 & 0,861 & 0,829 & 0,862 & 0,843 & 0,694 & 0,567 & 0,803 & 1,000 & & & & & & & & & & \\
\hline AP3 & 0,757 & 0,797 & 0,638 & 0,851 & 0,871 & 0,738 & 0,823 & 0,868 & 0,368 & 0,654 & 0,817 & 1,000 & & & & & & & & & \\
\hline M1 & 0,706 & 0,814 & 0,757 & 0,799 & 0,763 & 0,815 & 0,763 & 0,698 & 0,561 & 0,790 & 0,843 & 0,764 & 1,000 & & & & & & & & \\
\hline M2 & 0,784 & 0,799 & 0,689 & 0,774 & 0,741 & 0,847 & 0,763 & 0,638 & 0,617 & 0,852 & 0,876 & 0,749 & 0,791 & 1,000 & & & & & & & \\
\hline M3 & 0,786 & 0,844 & 0,781 & 0,802 & 0,752 & 0,878 & 0,767 & 0,702 & 0,627 & 0,789 & 0,846 & 0,769 & 0,909 & 0,827 & 1,000 & & & & & & \\
\hline TD1 & 0,698 & 0,826 & 0,668 & 0,851 & 0,870 & 0,720 & 0,865 & 0,748 & 0,439 & 0,702 & 0,849 & 0,833 & 0,865 & 0,762 & 0,821 & 1,000 & & & & & \\
\hline TD2 & 0,738 & 0,835 & 0,818 & 0,731 & 0,715 & 0,883 & 0,742 & 0,646 & 0,559 & 0,761 & 0,880 & 0,777 & 0,873 & 0,819 & 0,872 & 0,782 & 1,000 & & & & \\
\hline TD3 & 0,646 & 0,804 & 0,687 & 0,808 & 0,761 & 0,778 & 0,760 & 0,748 & 0,594 & 0,759 & 0,856 & 0,784 & 0,836 & 0,783 & 0,804 & 0,870 & 0,788 & 1,000 & & & \\
\hline GR1 & 0,791 & 0,861 & 0,707 & 0,774 & 0,819 & 0,722 & 0,777 & 0,758 & 0,423 & 0,719 & 0,859 & 0,866 & 0,802 & 0,741 & 0,785 & 0,834 & 0,838 & 0,752 & 1,000 & & \\
\hline GR2 & 0,705 & 0,782 & 0,798 & 0,808 & 0,770 & 0,864 & 0,783 & 0,615 & 0,488 & 0,764 & 0,881 & 0,790 & 0,881 & 0,852 & 0,830 & 0,842 & 0,852 & 0,845 & 0,761 & 1,000 & \\
\hline GR3 & 0,777 & 0,891 & 0,779 & 0,861 & 0,838 & 0,828 & 0,813 & 0,733 & 0,499 & 0,768 & 0,927 & 0,854 & 0,873 & 0,825 & 0,861 & 0,909 & 0,878 & 0,895 & 0,883 & 0,892 & 1,000 \\
\hline
\end{tabular}

Fonte: elaborada pelos autores (2018).

A planilha de dados padronizados com 98 casos (respondentes) e 20 variáveis, visto que a variável EP3 foi retirada, foi processada no software Statistica ${ }^{\circledR}$. Foi adotado o critério de Kaiser (1958) para extração inicial de fatores, ou seja, foram considerados como relevantes fatores com autovalores maiores que 1,0. Nesse sentido, foram identificados dois fatores iniciais. O Fator 1 explica aproximadamente $80 \%$ da variância e o fator 2, por sua vez, explica aproximadamente $5 \%$ da variância total dos dados. Os demais fatores isoladamente não explicam significativamente a variância. A Figura 2 apresenta a representação gráfica conhecida como scree plot com os autovalores de todos os vinte fatores iniciais. Observa-se que apenas dois fatores (F1 e F2) possuem autovalores maiores que 1,0. Nota-se ainda que o "salto" entre F1 e F2 é bastante elevado e ainda pode ser observado um "salto" ainda que menor entre F2 e F3. A partir de F3 o decréscimo dos autovalores são mínimos, o que demonstra que esses fatores explicam muito pouco a variância e não devem ser considerados. 


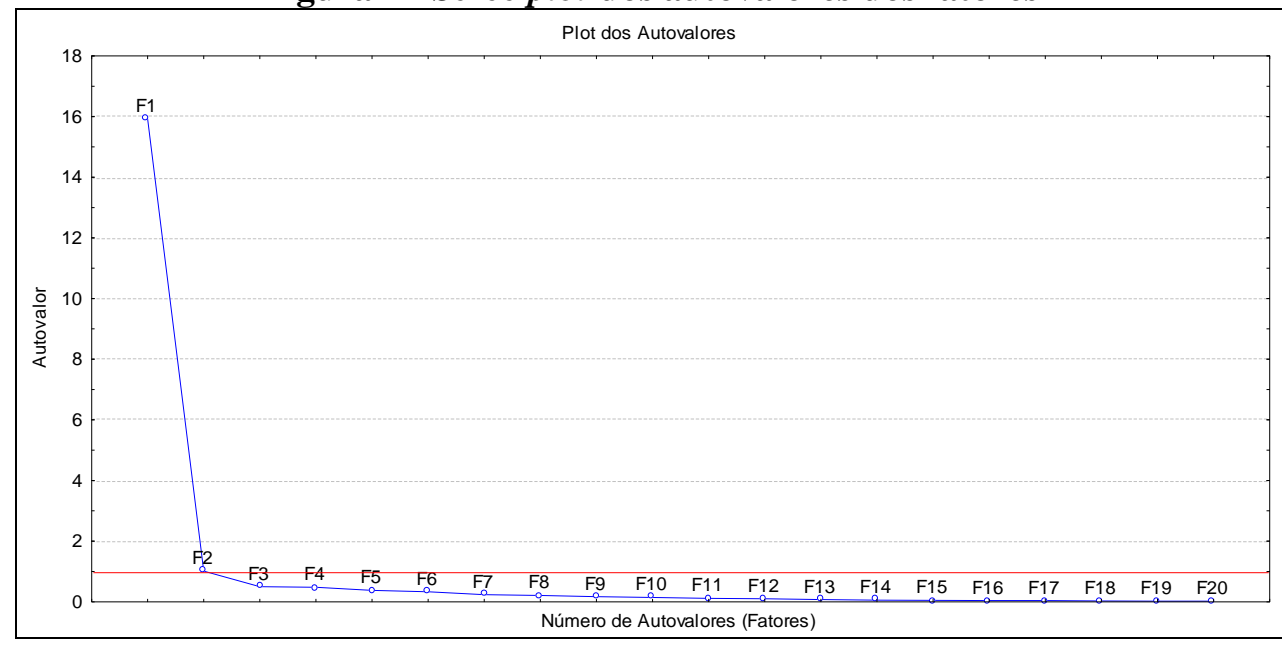

Fonte: elaborada pelos autores (2018).

A Tabela 3 demonstra as cargas fatoriais com a rotação obtida pelo método Varimax, considerando cargas fatoriais maiores que 0,65 como relevantes.

Tabela 3 - Cargas fatoriais obtidas após a rotação Varimax dos fatores

\begin{tabular}{ccc}
\hline & Fator 1 & Fator 2 \\
\hline FC1 & $\mathbf{0 , 6 7 8 3 5 5}$ & 0,515123 \\
FC2 & $\mathbf{0 , 6 9 8 1 1 6}$ & 0,594036 \\
FC3 & $\mathbf{0 , 8 4 6 0 0 7}$ & 0,267737 \\
L1 & 0,449315 & $\mathbf{0 , 8 2 9 3 3 7}$ \\
L2 & 0,383070 & $\mathbf{0 , 8 8 1 4 2 9}$ \\
L3 & $\mathbf{0 , 8 2 9 7 2 3}$ & 0,426244 \\
EP1 & 0,445550 & $\mathbf{0 , 8 1 5 4 5 5}$ \\
EP2 & 0,300269 & $\mathbf{0 , 8 4 0 9 5 4}$ \\
AP1 & $\mathbf{0 , 7 7 3 0 8 9}$ & 0,395372 \\
AP2 & $\mathbf{0 , 7 2 5 4 3 1}$ & 0,618764 \\
AP3 & 0,456171 & $\mathbf{0 , 8 2 0 6 4 2}$ \\
M1 & $\mathbf{0 , 7 3 7 5 1 3}$ & 0,555731 \\
M2 & $\mathbf{0 , 7 5 8 1 6 3}$ & 0,498255 \\
M3 & $\mathbf{0 , 7 7 2 3 7 9}$ & 0,525361 \\
TD1 & 0,538632 & $\mathbf{0 , 7 6 2 0 2 7}$ \\
TD2 & $\mathbf{0 , 8 1 7 3 4 3}$ & 0,462948 \\
TD3 & 0,628680 & 0,638876 \\
GR1 & 0,585381 & $\mathbf{0 , 6 9 0 1 0 1}$ \\
GR2 & $\mathbf{0 , 7 6 6 6 1 8}$ & 0,523393 \\
GR3 & $\mathbf{0 , 6 9 5 9 8 7}$ & $\mathbf{0 , 6 5 9 7 4 7}$ \\
Autovalor & 8,804499 & 8,142840 \\
Variância explicada & $44,0 \%$ & $40,7 \%$ \\
Variância total explicada & & $84,7 \%$ \\
\hline Font
\end{tabular}

Fonte: elaborada pelos autores (2018). 


\section{(1) SIIEF}

Após a rotação Varimax foi possível separar cargas fracas e fortes em cada um dos fatores, possibilitando uma análise mais precisa de cada fator. Segundo Hair et al. (2009, p.121), quando uma variável apresenta cargas fatoriais elevadas em ambos os fatores, ela é chamada de carga cruzada e deve ser desconsiderada da análise, pois não pode explicar cada fator isoladamente. Sendo assim, a variável GR3, que possui cargas cruzadas, ou seja, cargas elevadas em ambos os fatores, foi desconsiderada na análise de fatores.

Todas as variáveis associadas aos princípios de gestão "Foco no Cliente" e "Melhoria" e a maioria das variáveis associadas ao princípio "Abordagem de Processos ficaram associadas ao Fator 1. O Fator 2 incorporou todas as variáveis associadas ao "Engajamento das Pessoas" e a maioria das variáveis associadas ao princípio "Liderança", como demonstra a Figura 3.

Figura 3 - Fator 1 versus Fator 2, após a rotação pelo método Varimax.

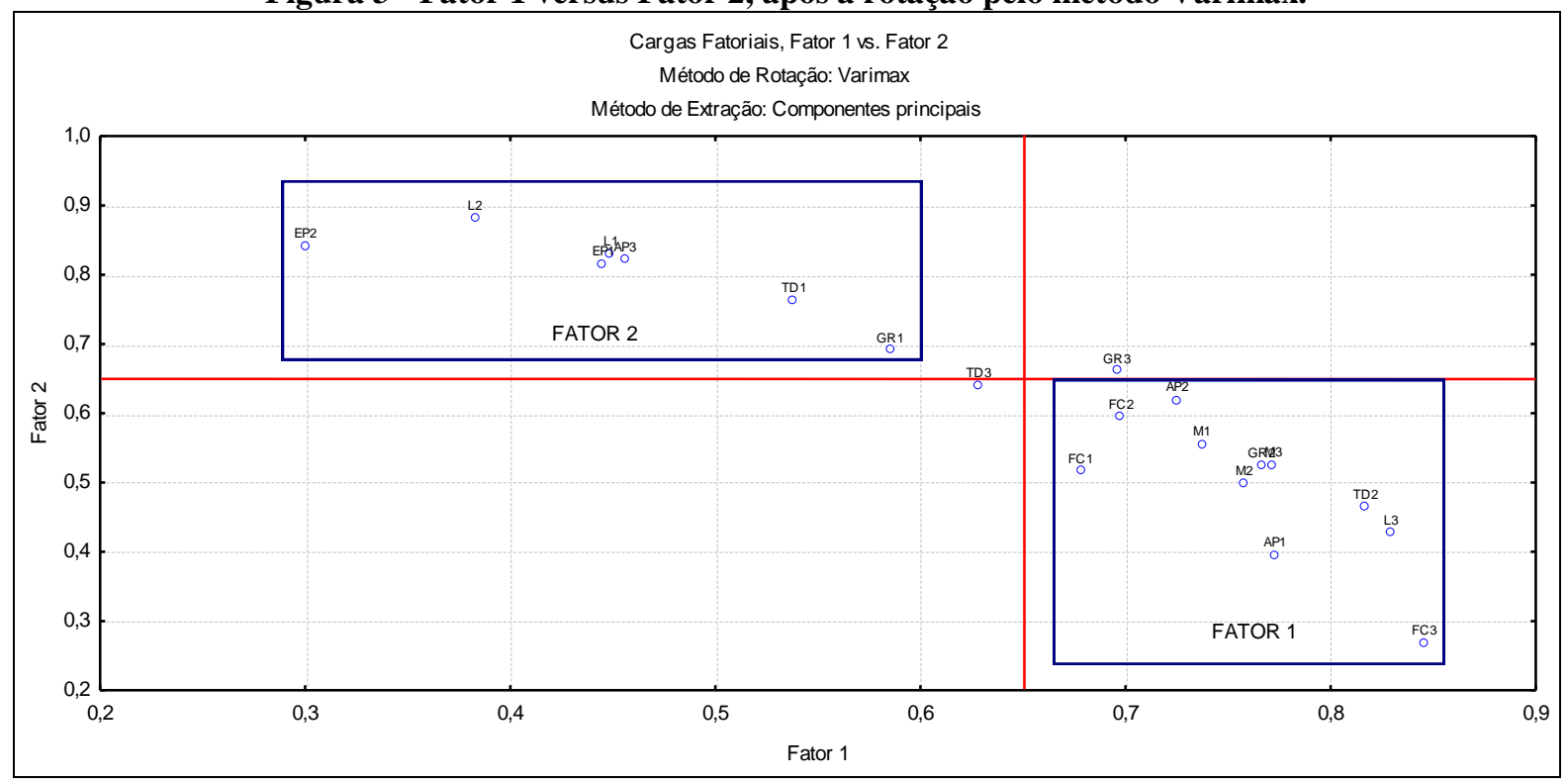

Fonte: elaborada pelos autores (2018).

As variáveis L3, TD2 e GR2 também incorporaram o Fator 1. Em relação à variável L3, ao comparar seu enunciado com as variáveis L1 e L2, observa-se que ela possui foco no ambiente para que as pessoas possam envolver-se, ao passo que L1 e L2 focam no estabelecimento e comunicação de estratégias. Percebe-se que L3 mensura mais o ambiente de trabalho que o sistema de liderança propriamente dito. TD2 considera a abordagem factual para tomada de decisões assertivas para a gestão dos processos. GR2 considera a existência de processos estruturados para a gestão do relacionamento. 


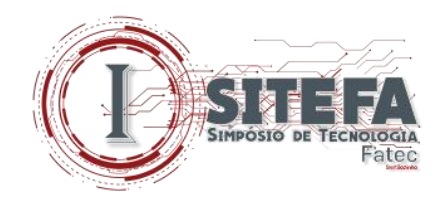

Segundo Hair et al. (2009, p.122), “...quando é obtida uma solução fatorial aceitável na qual todas as variáveis têm uma carga significante em um fator, o pesquisador tenta designar algum significado para o padrão de cargas fatoriais". Desse modo, como as variáveis com cargas mais elevadas no Fator 1 estão orientadas aos princípios de gestão "Abordagem de Processo", "Foco no Cliente" e "Melhoria", o Fator 1 pode ser denominado como Gestão de Processos. Além disso, as variáveis L3, TD2 e GR2 também podem ser associadas à gestão de processos.

O Fator 2, por sua vez, incorpora além das variáveis já mencionadas, as variáveis AP3, TD1, GR1 e GR3. Como GR3 possui carga cruzada entre os dois fatores, não foi considerada na análise multivariada. AP3 investigou as capacidades dos processos serem analisados por meio de medição e análise e, para o desenvolvimento dessas análises é fundamental que as pessoas possuam competências para tais análises. TD1 procura investigar se as pessoas possuem acesso a informações e ferramentas para análise de dados para a tomada de decisões. A variável GR1 aborda o relacionamento entre a organização e parceiros. Sendo assim, como as variáveis com cargas mais elevadas no Fator 2 estão mais direcionadas aos princípios de gestão "Liderança" e "Engajamento das Pessoas", este fator pode ser denominado como Gestão de Pessoas. As variáveis AP3, TD1 e GR1, por sua vez, também podem ser associadas à gestão de pessoas.

\section{CONCLUSÃO}

Este trabalhou procurou investigar como os princípios de gestão da qualidade estabelecidos na norma ISO 9000:2015 e reproduzidos na norma ISO 9001:2015 estão sendo implementados e quais os benefícios resultantes dessa implementação pela perspectiva dos empregados. A preocupação com as práticas de gestão da qualidade para aumentar a competitividade organizacional e a satisfação dos clientes tem aumentado ao longo do tempo, visando que a organização promova ações estratégicas para alcançar uma posição de destaque no ambiente competitivo globalizado (GOHR; FAUSTINO, 2017).

Sendo assim, uma organização industrial certificada ISO 9001 envolvida na cadeia de suprimentos sucroenergética foi selecionada para ser o objeto do estudo de caso. No intuito de captar a percepção dos empregados quanto à percepção do grau de implementação dos 


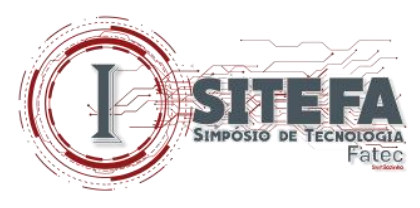

princípios de gestão da qualidade, foi elaborado um instrumento de coleta de dados baseado no questionário proposto na norma ABNT NBR ISO 10014:2008 (ABNT, 2008)

Para validar o instrumento de coleta de dados, uma primeira versão desse instrumento foi apresentada para um grupo de doze respondentes de áreas de atuação, tempos de casa e cargos distintos na organização. Posteriormente foi cálculo coeficiente $\propto$ de Cronbach para testar a consistência interna do questionário, sendo obtido um coeficiente $\propto=0,94$, o que foi considerado satisfatório para o instrumento de coleta de dados.

Os dados obtidos foram processados por softwares específicos para realização das análises estatísticas dos dados. Primeiramente foi realizada a análise descritiva dos dados. Conclui-se que por uma abordagem univariada dos dados, ou seja, com as variáveis sendo analisadas isoladamente, o princípio de gestão "Foco no Cliente" possui maior nível de implementação pela perspectiva dos empregados, sendo que o princípio de gestão "Liderança" possui o menor nível de implementação, o que pode levar a sugestão de que ações prioritárias devem ser implementadas em função desse princípio de gestão.

Partindo do pressuposto que os princípios de gestão da qualidade foram estabelecidos de forma inter-relacionada, a pesquisa buscou investigar o grau de inter-relações existentes entre esses princípios por meio de técnicas de análise multivariada dos dados, mais especificamente a técnica conhecida como análise de fatores por componentes principais.

As técnicas estatísticas multivariadas levam à simplificação de fenômenos mensuráveis para poucas novas variáveis latentes, ou seja, que não podiam ser medidas diretamente. Entre as vantagens gerenciais dessas técnicas estão a simplificação de múltiplas variáveis para poucas variáveis, onde o gestor pode direcionar melhor suas ações, de forma mais assertiva e mais eficiente, minimizando recursos e esforços em processos de melhoria.

Essa análise reduziu o número inicial de vinte e uma variáveis para dois fatores ortogonais distintos que carregaram cargas fatoriais dessas variáveis originais. Esses dois fatores foram capazes de explicar a variância de aproximadamente $85 \%$ dos dados originais, o que é muito relevante.

O primeiro fator foi denominado de gestão de processos, pois carregava cargas elevadas de variáveis originais associadas a processos, com predominância das variáveis associadas aos princípios de gestão "Abordagem de Processos", "Foco no Cliente" e 


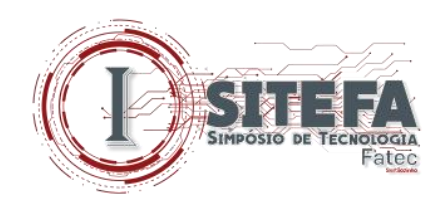

"Melhoria". O segundo fator, denominado gestão de pessoas, carregou cargas fatoriais fortes predominantemente dos princípios "Liderança" e "Gestão de Pessoas".

Com esses fatores, que até então eram latentes, a organização pode focar seus esforços de melhoria em duas dimensões: processos e pessoas, o que simplifica a implementação de ações após uma análise univariada dos dados, onde a organização deveria promover ações específicas para melhoria de cada um dos princípios de gestão da qualidade, iniciando-se por "Liderança" que foi o pior avaliado.

Entende-se assim que os objetivos da pesquisa foram plenamente alcançados, demonstrando que a metodologia proposta na pesquisa foi adequada levando a obter resultados relevantes para a organização.

\section{REFERÊNCIAS}

ARANHA, Francisco; ZAMBALDI, Felipe. Análise fatorial em administração. São Paulo: Cengage Learning, 2008.

ASSOCIAÇÃO BRASILEIRA DE NORMAS TÉCNICAS (ABNT). ABNT NBR ISO 9000: sistemas de gestão da qualidade: fundamentos e vocabulário. Rio de Janeiro, 2015a.

$2015 b$.

ABNT NBR ISO 9001: sistemas de gestão da qualidade: requisitos. Rio de Janeiro,

ABNT NBR ISO 9004: gestão para o sucesso sustentado de uma organização: uma abordagem da gestão da qualidade. Rio de Janeiro, 2010.

ABNT NBR ISO 10014: Gestão da qualidade: diretrizes para a percepção de benefícios financeiros e econômicos. Rio de Janeiro, 2008.

COMITÊ BRASILEIRO DA QUALIDADE (CB-25). ABNT/CB-025: Comitê Brasileiro da Qualidade. Disponível em: 〈http://www.abnt.org.br/cb-25>. Acesso em: 14 dez. 2017.

CRONBACH, Lee. Coefficient alpha and the internal structure of tests. Psychometrika, v.16, n.3, p.297-334, 1951.

DENTON, Paul David; MAATGI, Musbah Kharis. The development of a work environment framework for ISO 9000 standard success. International Journal of Quality \& Reliability Management, Bingley, v.33, n.2, p.231-245, oct. 2016.

FÁVERO, Luiz Paulo; BELFIORE, Patrícia. Análise de dados: técnicas multivariadas exploratórias com SPSS® e STATA®. Rio de Janeiro: Elsevier, 2015. 


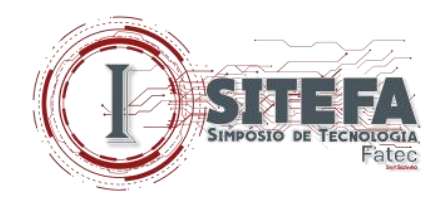

FONSECA, Luís; DOMINGUES, José Pedro. ISO 9001:2015 edition: management, quality and value. International Journal for Quality Research, Kragujevac, v.11, n.1, p.149-158, mar. 2017.

GOHR, Cláudia Fabiana; FAUSTINO, Cinthia de Azevêdo. Gestão da Qualidade na Cadeia de Suprimentos. Revista Pretexto, Belo Horizonte, v.18, n.4, p.33-56, out.-dez. 2017.

HAIR, Joseph F. et al. Análise multivariada de dados. 6.ed. Porto Alegre: Bookman, 2009.

INTERNATIONAL ORGANIZATION FOR STANDARTZATION (ISO). About ISO.

Disponível em: <https://www.iso.org/about-us.html>. Acesso em: 09 dez. 2017.

KAISER, Henry F. The varimax criterion for analytic rotation in factor analysis.

Psychometrika. v.23, n.3, p.187-200, sep. 1958.

LATTIN, James; CARROLL, J. Douglas; GREEN, Paul E. Análise de dados multivariados. São Paulo: Cengage Learning, 2011.

LOESCH, Claudio; HOELTGEBAUM, Marianne. Métodos estatísticos multivariados. São Paulo: Saraiva, 2012.

MAROCO, J.; GARCIA-MARQUES, T. Qual a fiabilidade do alfa de Cronbach? Questões antigas e soluções modernas? Laboratório de Psicologia, Lisboa, v.4, n.1, p.65-90, jul. 2006.

MORADI, Tayebeh et al. Quality management systems implementation compared with organizational maturity in hospital. Global Journal of Health Science, Toronto, v.8, n.3, p.174-182, jul. 2015.

RUSJAN, Borut; ALIČ, Milena. Capitalising on ISO 9001 benefits for strategic results. International Journal of Quality \& Reliability Management, Bingley, v.27, n.7, p.756778 , jan. 2010.

SINGH, Prakash J.; SMITH, Alan. An empirically validated quality management measurement instrument. Benchmarking: An International Journal, Bingley, v.13, n.4, p.493-522, 2006.

TOLEDO, José Carlos de et al. Qualidade: gestão e métodos. São Paulo: LTC, 2012.

WOLNIAK, Radoslaw. The assessment of significance of benefits gained from the improvement of quality management systems in Polish organizations. Quality \& Quantity, Dordrecht, v.47, n.1, p.515-528, jul. 2011. 